\title{
QoS guarantee protocol based on combination of opportunistic dynamic cloud service and cooperative multimedia stream for wireless sensor networks
}

\author{
Yong Jin', Ruigang Li ${ }^{2}$, Huan Dai ${ }^{i^{*}}$ and Junchao Zhang ${ }^{3}$
}

\begin{abstract}
In real-time multimedia applications, the most problems are how to store, process, and transport the multimedia data with cloud, how to optimize the cloud platform to satisfy the user demand, as well as how to provide the quality of service (QoS) guarantees by combining cloud platform and wireless sensor networks (WSNs). To overcome these issues, the multimedia cloud was designed according to the characteristics of group of pictures (GOP), determining degree, and video frame density. The opportunistic dynamic multimedia cloud platform was proposed on the basis of the variation of packet error rate, decodable frame ratio and peak signal-to-noise ratio (PSNR) with channel quality. Then, the optimal multi-relay hierarchical cooperative multimedia transmission scheme was designed and implemented by reconstructing the cooperative multimedia stream based on the framing of GOP. For enhancing the multimedia cloud computing efficiency and decreasing the complexity of encoding and decoding of multimedia stream, we combined the opportunistic dynamic multimedia cloud platform and cooperative multimedia stream, which can also enhance the resources utilization of WSNs. Finally, the QOS guarantee protocol with the combination of opportunistic dynamic multimedia cloud platform and cooperative multimedia stream for WSNs (ODM-CM-WSN) was presented. Especially, the layered architectural model for multimedia applications and the opportunistic cooperative multimedia cloud service model were developed. The mathematical and simulation results demonstrate that the proposed QoS guarantee protocol can satisfy the various requirements of multimedia applications and provide reliable and efficient cloud services with WSNs.
\end{abstract}

Keywords: Multimedia cloud; Opportunistic cloud platform; Cooperative multimedia stream; Wireless sensor networks; QoS guarantee

\section{Introduction}

With the wide use of Internet, Internet of Things, and cloud computing technologies $[1,2]$, the rapid development of distributed computing and sensing technologies, intelligent perception, interaction, and communication is affecting the development process of human life and society. In the meantime, multimedia applications have gradually become an important application finance, manufacturing, telecommunications, retail, and other fields $[3,4]$, which will become one of the largest fields

\footnotetext{
*Correspondence: daihuanjob@163.com

'School of Computer Science \& Engineering, Changshu Institute of

Technology, Changshu 215500, China

Full list of author information is available at the end of the article
}

of the cloud services market. However, there are some issues such as third party attacks [5], data leakage [6], decreasing of quality of experience, and difficulty of meeting the user demand of diversity in various applications. Emerging cloud computing platform service reliability and data communications stability have been concerned and studied by more and more researchers. Specially, the cloud platform is used to provide services of data storage and management and difficult to provide protection for the process of data transfer and sensing the quality of experience, etc. So Alamri et al. [7] proposed the architecture, applications, and approaches of sensor-cloud. Zeng et al. [8] proposed the abnormal vehicles-oriented wireless sensor networks (WSNs)-cloud 
infrastructure, which is able to provide safety and comfort for drivers and improve environmental conditions.

First, mobile cloud computing (MCC) [9] has been the important development direction of cloud computing and cloud service support platform, which will play one important role in various domains such as mobile learning, electronic commerce, medical and health work, as well as social network. The framework of energy-efficient location-based service and mobile cloud convergence was proposed by Choi et al. [10], which substituted the powerintensive sensors for decreasing the power dissipation. By researching how to minimize the energy consumption of displaying a video stream, Lin et al. [11] proposed the algorithm for resolving the fundamental problem and proving the optimality in terms of energy savings, as well as without impacting the user's visual experience. By removing redundant and non-informative frames, Mehmood et al. [12] presented the wireless capsule endoscopy video summarization framework, which could improve the resizable computing capacities between the client and the cloud server.

Second, Farooqi et al. [13] proposed the novel intrusion detection framework, which applied to the distributed environment for detecting intrusion. The trusted big data capture and transport architecture were constructed by Zhou et al. [14] via discussing and designing the concepts of big data, trust and reputation, and WSNs. Through combining the cloud-based wireless body area networks with statistical modeling techniques, the new architecture was proposed by Diallo et al. [15], which was used to provide a secure storage infrastructure and optimize the real-time user query processing. Li et al. [16] developed the attenuation distributed approximating approach for fundamental, harmonic and interharmonic frequency, and phasor estimation during energy measurement, which applied to any form of signal.

Especially, Shamshirband et al. [17] summarized and classified the intrusion detection and prevention systems (IDPS) schemes according to the traditional artificial computational intelligence with a multi-agent support. Garcia-Valls et al. [18] studied the challenges about supporting the real-time applications with the cloud and cloud computing technology. Kourtesis et al. [19] overviewed the main protocols about semantic-based quality of service (QoS) management and the principal methods for processing and exploiting diverse data and advanced real-time monitoring services. The channel characterization scheme was proposed by Mendes et al. [20], which combined to a cross-layer admission control in dynamic cloudbased multimedia sensor networks for sharing the network resources. An activity recognition application was developed, and the transmission protocol of the data to the cloud for further processing was studied by Hussain et al. [21]. Misra and Chatterjee [22] studied the fundamental issues in context aggregation of health data within the mobile monitoring nodes and researched the channelization of the aggregated data by dynamic selection of the cloud gateways. By identifying key characteristics of the dynamic systems, Dautov et al. [23] studied the cloud application platforms to distributed sensor networks and analyzed the viability of exploiting the similarities.

For resolving the issues such as the authentication, trust, and reputation calculation of cloud service providers and sensor network providers, the novel authenticated trust calculation and management system for cloud computing-WSNs integration was proposed by Zhu et al. [24]. Ren and van der Schaar [25] have employed the cloud-based stream mining system in the mobile devices for sending unclassified media streams to the cloud for classification via wireless links.

However, there are some problems in the above researches, which are as follows: (1) How to create the cloud for multimedia data storage, processing, and transmission; (2) How to build the optimal cloud platform based on user demand; (3) How to establish the optimal mapping and transmission mode between cloud platforms and WSNs; and (4) How to make the QoS guarantees for multimedia communications based on the combination of cloud platform and WSNs.

To solve these problems, the main contributions of our work are as follows: (1) multimedia cloud was designed, which sustained the passive tags, active on-demand services, heterogeneous network compatibility, and resource sharing of multimedia streaming; (2) opportunistic dynamic multimedia cloud platform was built according to the characteristics of multimedia cloud scales, signalto-noise ratio (SNR), decodable frame number, bit error rate, etc.; (3) reconstruction of cooperative multimedia streaming based on partial frame characteristics of the group of pictures (GOP); and (4) combining the opportunistic dynamic multimedia cloud platform and cooperative multimedia streaming to provide the QoS guarantee.

\section{Opportunistic dynamic multimedia cloud platform}

Let $M$ denote the quantitative collection of multimedia data. Let $T$ denote the multimedia streaming qualitative parameters collection of $M$ and $x$ denote the quantitative multimedia data. The determining degree $f(x)$ belongs to the interval $[0,1]$ and is a random number with a stable trend when the quantitative multimedia data $x$ belongs to $M$ and is the random mapping of $T$. Hence, multimedia cloud $M(x)$ is defined as the distribution of $x$ on multimedia domain $M$.

The $t$ video frames are stored in the $M(x)$, which defined as $F=F_{1}, F_{2}, \ldots F_{t}$. Each video frame belongs to intra-coded frame (I frame), forward predictive-coded frame (P frame), and bidirectional predictive-coded frame interpolation 
(B frame). The candidate video frame $F_{i}$ could be calculated by formula (1) based on user demand $U_{r}$ and $T$.

$$
F_{i}=\left\{\begin{array}{c}
T_{p}\left(F_{i}\right) \\
h\left(F_{i}, T\right) \\
\varphi\left(T_{p}, f\left(F_{i}\right) h\left(F_{i}, T\right), U_{r}\right)
\end{array} 0<i \leq t\right.
$$

Here, let $h\left(F_{i}, T\right)$ denote the analysis function of video frame with the qualitative parameters. Let $\varphi\left(T_{p}, f\left(F_{i}\right) h\left(F_{i}\right.\right.$, $T), U_{r}$ ) denote the combination of the above analysis results and user demand. Specially, combination of the determine degree $f\left(F_{i}\right)$ of video frames and qualitative analysis $h\left(F_{i}, T\right)$ could accurate the user mapping flow data.

Specially, let $T_{p}\left(F_{i}\right)$ denote the priority determined by video frame type. The video frame type contained in the multimedia stream could be analyzed according to the calculation results, which are as follows:

(1) The multimedia stream only contain I frame when $T_{p}\left(F_{i}\right)=1$, which would be defined the primary multimedia streaming $S_{0}$;

(2) The multimedia stream contain I frame and P frame when $T_{p}\left(F_{i}\right)=2$, which would be defined the secondary multimedia streaming $S_{L}$;

(3) The multimedia stream contain I frame, $P$ frame, and $\mathrm{B}$ frame when $T_{p}\left(F_{i}\right)=3$, which would be defined the advanced multimedia streaming $S_{H}$.

In addition, the priority of I frame is superior to one of $\mathrm{P}$ and $\mathrm{B}$ frames. The priority of $\mathrm{P}$ frame is larger than one of that of B frame.

There are the following relationships between determining degree and three-level multimedia stream.

$$
f(F)=T_{p}\left(F_{i}\right) \exp \left(-\frac{L\left(S_{0}\right)}{L\left(S_{L}\right)+L\left(S_{H}\right)}\right)
$$

Here, let $L\left(S_{0}\right), L\left(S_{L}\right)$, and $L\left(S_{H}\right)$ represent the length of $S_{0}, S_{L}$, and $S_{H}$, respectively, units of which are the number of packets. They have the following characteristics:

(1)Passive tag (uplink): WSNs upload the multimedia data and adaptive mark a cloud service with the establishment of a straight-through session;

(2)Active demand service (downstream): sending or forwarding multimedia data based on user needs WSNs actively;

(3) Heterogeneous network compatibility: heterogeneous networks compatible communication such as wired and wireless LAN, WSNs, Internet of Things (IOT), etc. The transparent processing module would be established between the above heterogeneous networks and cloud services.
(4) Sharing streaming cloud computing resource: to provide users with a long steady stream data service by the adaptive selection of multimedia cloud.

(5)Cloud service reliability: to provide users with a high reliability, real-time, and robust multimedia streaming service.

In the process of multimedia communication, multimedia cloud platform status could be defined as $\left\{N_{M}\right.$, SNR, $\left.N_{\text {dec }}, N_{\text {packet }}, P_{b}\right\} . N_{M}$ is the scale of multimedia cloud. Let $N_{\mathrm{dec}}$ denote the decodable frame number. Let $N_{\text {packet }}$ denote the packet number of the multimedia stream. $P_{b}$ is the bit error rate. When the multimedia clouds are independent and its distribution is Gaussian, the receiver SNR could be calculated by formula (3).

$$
\left\{\begin{array}{c}
S N R=g\left(F_{i}\right) \\
f(x)=\frac{E_{N}}{d} \sum_{i=1}^{N_{M}}\left[\left(2 N_{\text {packet }}-1\right) P_{b}\right] x
\end{array}\right.
$$

Here, $E_{N}$ is the Gaussian expectation. Let $d$ denote the distance between sender and receiver.

The value of $N_{\text {dec }}$ could be obtained by formula (4).

$$
\begin{aligned}
N_{\text {dec }} & =T_{p}\left(F_{i}\right) N_{\text {packet }}\left[\left(1-P_{b}\right)^{N_{I}}+P_{b}{ }^{N_{I}} \sum_{j=1}^{N_{P}}\left(1-P_{b}\right)\right. \\
& \left.+P_{b}{ }^{N_{I}+N_{P}} \sum_{j=1}^{N_{B}}\left(1-P_{b}\right)\right]
\end{aligned}
$$

Here, $N_{I}, N_{P}$ and $N_{B}$ are the number of I frame, P frame, and B frame, respectively.

The packet error rate could be calculated by formula (4).

$$
\left\{\begin{array}{c}
P_{M}=r\left(N_{\text {packet }}, N_{M}\right) \\
r(x, y)=1-\left(1-\frac{1}{2} e^{f\left(F_{i}\right)}\right) \frac{x / y}{x}
\end{array}\right.
$$

The value of peak signal-to-noise ratio (PSNR) could be obtained by formula (6).

$$
\text { PSNR }=20 \ln \left[\frac{N_{\text {dec }}}{N_{\text {packet }}} \frac{1}{\sqrt{\sum_{i=1}^{N_{I}} \sum_{j=1}^{N_{P}} \sum_{k=1}^{N_{B}}\left[C_{R}(I, P, B)-C_{S}(I, P, B)\right]}}\right]
$$

Here, assume $C_{S}$ and $C_{R}$ are the multimedia streaming structure at sender and receiver.

According to formulas (4), (5), and (6), we analyze and evaluate the performance of packet error rate, decodable frame ratio, and PSNR with bit error rate, which are illustrated in Figs. 1, 2, and 3. 


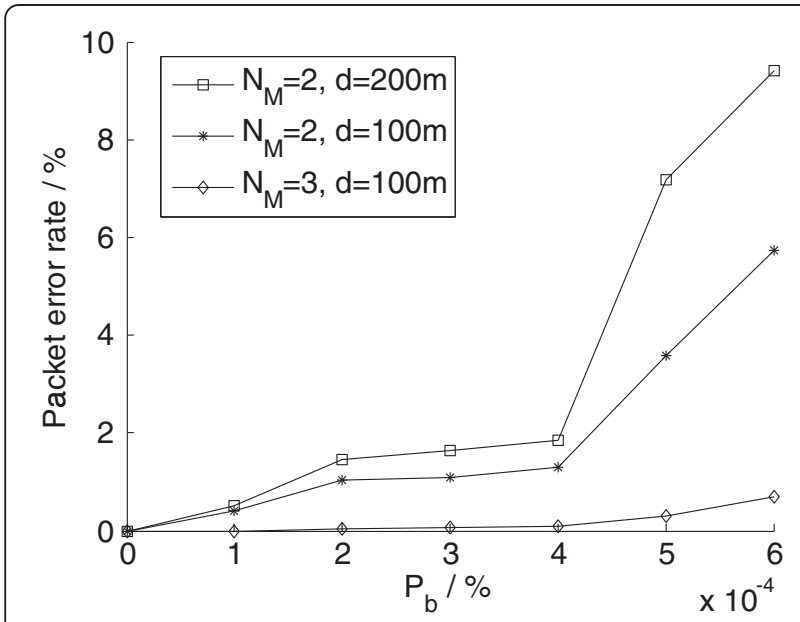

Fig. 1 Packet error rate with $P_{b}$

As shown in Fig. 1, when $P_{b}$ is increasing, packet error rate is increasing too. When multimedia cloud scale or distance is increasing, packet error rate is increasing. When channel quality is good and $N_{M}$ is 3 , packet error rate is close to zero. At this time, increasing the scale of multimedia cloud would not enhance performance. Figure 2 shows that the larger the $P_{b}$, the lower the decodable frame ratio. When multimedia cloud scale or distance is increasing, decodable frame ratio is increasing. When $P_{b}$ is larger than $3 \%$ and less than $4 \%, N_{M}$ and $d$ have less impact on SNR. If $P_{b}$ is larger than $4 \%$, the remote communication decreased video quality. The PSNR jitter could be smoothed by increasing multimedia cloud scale, which is beneficial to control. The above conclusions are illustrated in Fig. 3.

Based on the analytical result of packet error rate, decodable frame ratio, and PSNR, opportunistic dynamic multimedia cloud platform can be built in the following steps:

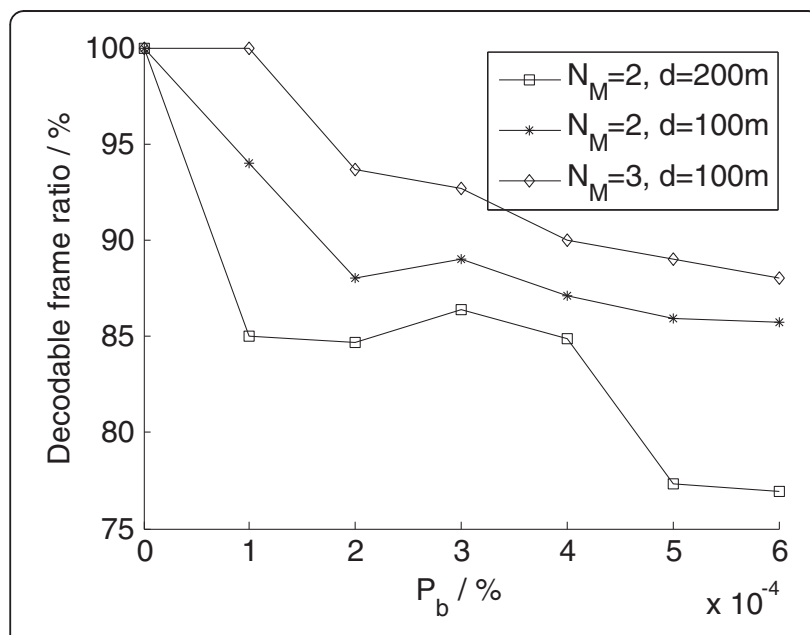

Fig. 2 Decodable frame ratio with $P_{b}$

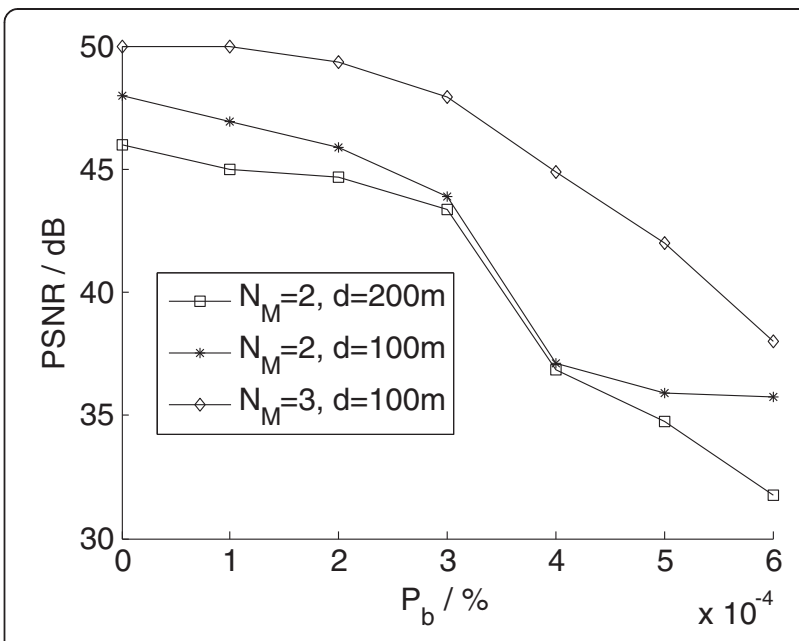

Fig. 3 PSNR with $P_{b}$

Step 1: Multimedia streaming based on MPEG encoded algorithm is defined as "I frame, P frame, and B frame." Multimedia cloud state is defined as "remaining storage space $\mathrm{SR}_{i}$, communication distance $d_{c}$, the transmission power $P_{S}$." Here, parameter values of multimedia streaming status can be obtained after system initialization. Parameter value of multimedia cloud state can be obtained based on the inherent properties of the cloud equipment.

Step 2: Calculating the candidate video frame $F_{i}$ based on the combination of user demand $U_{r}$ and $T$ according to the parameter values of step 1 .

Step 3: Combining the determining degree of video frame $F_{i}$ and qualitative analysis result of $h\left(F_{i}, T\right)$ and then making the mapping between user and multimedia stream collection.

Step 4: Deciding the frame type $T_{p}\left(F_{i}\right)$ of multimedia stream. If there are only I frames in multimedia stream, $T_{p}\left(F_{i}\right)$ is set to 1 . If there are only I frames and $\mathrm{P}$ frames in multimedia stream, $T_{p}\left(F_{i}\right)$ is set to 2 . If there are I frames, $\mathrm{P}$ frames, and $\mathrm{B}$ frames in multimedia stream, $T_{p}\left(F_{i}\right)$ is set to 3 . Making the relationship between determining degree and three-level multimedia stream.

Step 5: The state of the multimedia cloud platform is defined as " $\left\{N_{M}, \mathrm{SNR}, N_{\text {dec }}, N_{\text {packet }}, P_{b}\right\}$." When $N_{M}$ and $d_{c}$ are regular, the influence law of decodable frame ratio with $P_{b}$ could be obtain according to the formula (4). Similarly, the influence law of packet error rate with $P_{b}$ could be obtained according to the formula (5). The influence law of PSNR with $P_{b}$ could be obtained according to the formula (6).

Step 6: If the user focuses on the reliable quality of service guarantee, the step 7 should be carried out. If the user focuses on playable quality of service guarantee, the step 8 should be carried out. If the user 
wants to guarantee the performance of dynamic cloud platform multimedia, the step 9 should be carried out. Step 7: Decreasing the packet error rate by increasing multimedia cloud scale and $d$ is not too large. When $P_{b}$ is small and $N_{M}$ is 3, packet error rate is close to zero. So, adding new multimedia clouds is unnecessary. Step 8: Improving the PSNR by increasing $N_{M}$ and decreasing $d$ because $P_{b}$ is inversely proportional to $d_{c}$. Step 9: Reducing the value of $N_{M}$ and $d_{c}$ when $P_{b}$ is between 3 and $4 \%$. $N_{M}$ should be increased to smooth PSNR jitter of remote communication when $P_{b}$ is larger than $4 \%$.

\section{Cooperative multimedia streaming based on the framing of GOP}

There are $n$ video frames, $m$ GOP and $k$-type frame in the multimedia stream. The throughput with WSNs could be obtained by formula (7).

$$
T(n) \leq \frac{\sum_{l=1}^{N_{R}} \sum_{i=1}^{m} \sum_{j=1}^{k} \varphi\left(F_{m}\right)}{\sum_{p=1}^{n} \varphi\left[(\alpha+\beta+\gamma) F_{p}\right]}
$$

Here, $N_{R}$ is the scale of cooperative sensors. Let $\varphi\left(F_{m}\right)$ denote the video frame, which could be decoded at the receiver correctly. As illustrated in formula (7), $T(n)$ is proportional to $N_{R}$ and number of GOP, which is inversely proportional to the video frame type. So, the multi-relay-grade cooperation transmission scheme of multimedia streaming has the following advantages:

(1)Obtain the more spatial diversity gain through multi-relay transmission;

(2) Guarantee real-time performance by optimizing the flow of the frame structure, the size of the compressed stream for energy gain;

(3) Obtain long-distance transmission reliability guarantee with the multi-relay-grading.

So, the multimedia stream would be reconstructed as " $n, m$, and $k$ " according to the above proposed opportunistic dynamic multimedia cloud platform, which would be transferred to $N_{R}$ relay sensors. Feature of I, P, and $\mathrm{B}$ frames should be defined as " $\alpha, \beta, \gamma$." The hierarchical cooperative transmission network is constructed by $\left\{S, N_{R}, D\right\}$. Here, $D$ is the destination sensor of the multimedia stream.

When the relay sensors and $D$ sensor received the multimedia stream successfully, the multimedia stream would be reconstructed. Then, the acknowledge packet would be transferred to $S$ sensor.
The following issues should be paid more attention when the multimedia stream transmitted by the hierarchical cooperative transmission network.

(1) If the feedback of $D$ sensor is consistent with feedback from $N_{R}$ relay sensors, the multimedia stream have been received correctly.

(2) If not, the inconsistent relay sensors have to leave the hierarchical cooperative transmission network.

(3) The failed relay sensor should send the failure multimedia data packets to the neighbor sensors. The candidate relay sensor would be found when the neighbor sensors and $D$ sensor received the packets, which would take the place of the failed sensor.

(4) The process of (3) could guarantee the stability of the hierarchical cooperative transmission network and transmitter the failed packets to $D$ sensor by one retransmission.

There are three phases in the progress of constructing the hierarchical cooperative transmission network, which are as follows:

The first stage: to establish the collection of hierarchical cooperative sensors

The receiving power $P_{r}$ of $D$ sensor could be obtained by formula (8). Here, $P_{1}$ is the receiving power of relay sensor l, which could be calculated by formula (9).

$$
\begin{aligned}
& P_{r}=\sum_{l=1}^{N_{R}} P_{l}\left(\frac{d}{d_{(S, l)}}\right)^{-\rho} 2 \leq \rho \leq 5 \\
& P_{l}\left(N_{R}\right)^{l}=P\left(\frac{d}{d_{(S, l)}}\right)^{-\rho} 2 \leq \rho \leq 5
\end{aligned}
$$

Here, $\left(N_{R}\right)^{1}$ denotes the 1 relay sensor in the set of relay sensors.

For the parallel communication, $P_{S}$ have to be smaller than $P_{r}\left(m^{\alpha} m^{\beta} m^{\gamma}\right) / n$. Under the extreme conditions, multi-relay SNR of hierarchical cooperative network is illustrated by formula (10).

$$
S_{N_{R}}=\frac{\sum_{l=1}^{N_{R}+1} P_{r} \frac{m^{\alpha+\beta+\gamma}}{n}}{\sum_{l=1}^{N_{R}+1} P_{l}\left(\frac{d}{d_{(S, l)}}\right) P_{r}\left(N_{R}\right)^{l}}=\frac{d^{-\rho}}{\sum_{l=1}^{N_{R}+1} d_{(s, l)}-\rho}
$$

The number of channels for transmitting multimedia stream could be calculated by formula (11).

$$
C_{N}=\frac{n}{m\left(N_{R}+2\right)^{\alpha+\beta+\gamma}} N_{M}^{k}
$$

Here, let $N_{M}^{k}$ denote that $k$ clouds were selected from $M$. 
The decodable frame ratio DFR could be calculated by formula (12).

$$
\begin{aligned}
D F R & =\left\{E\left[e^{\alpha}\right] E\left[e^{\beta}\right] E\left[e^{\gamma}\right]\right\} \bar{m} e^{-N_{R} N_{M}} \frac{d^{-\rho}}{\sum_{l=1}^{N_{R}} d_{l}^{-\rho}} \\
& \leq e^{-N_{R} N_{M}} d^{-\alpha}
\end{aligned}
$$

The following conditions should be considered:

(1)Parallel communication and limited interference between sensors

(2) Maximize the number of channels

(3) Maximize the decodable frame ratio of $D$ sensor

On the basis of the above constraints and formulas, we can obtain the optima value of $N_{R}$ and $N_{M}$, which is illustrated in Fig. 4. In Fig. 4, we divided the network to $6 * 5$ matrix. There are four multimedia clouds and three relay sensors in the grid.

The second stage: multi-relay cooperative transmission.

Each relay sensor and $S$ sensor obtains the multimedia stream from the mapping multimedia clouds. Then, the multimedia stream would be sent to $D$ sensor after reconstruction as " $n, m$, and $k$ " and encoding independently. The parallel sending power have to be smaller than $P_{r(S, D)}^{\rho} \frac{m}{n}$.

The multimedia stream would be reconstructed only without decoding. The number of transmission frames belongs to the interval $\left[\frac{m}{n}, n\right]$. The time of hierarchical cooperative transmission is equal to $2 N_{R} N_{M}$. The multirelay cooperative transmission topology is shown in Fig. 5. Figure 5 shows that the opportunistic dynamic multimedia cloud platform would be established according

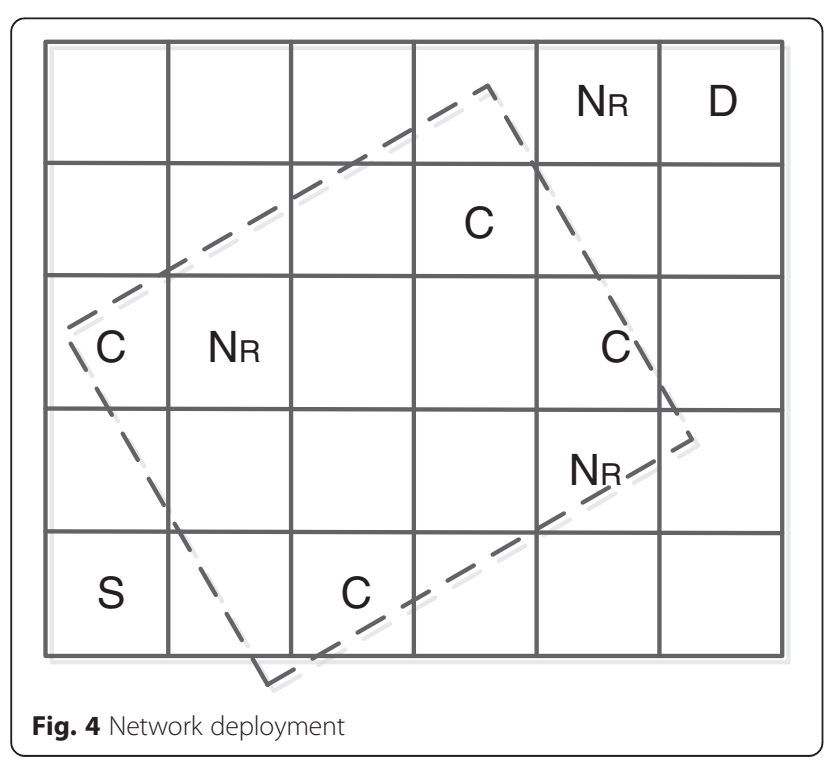

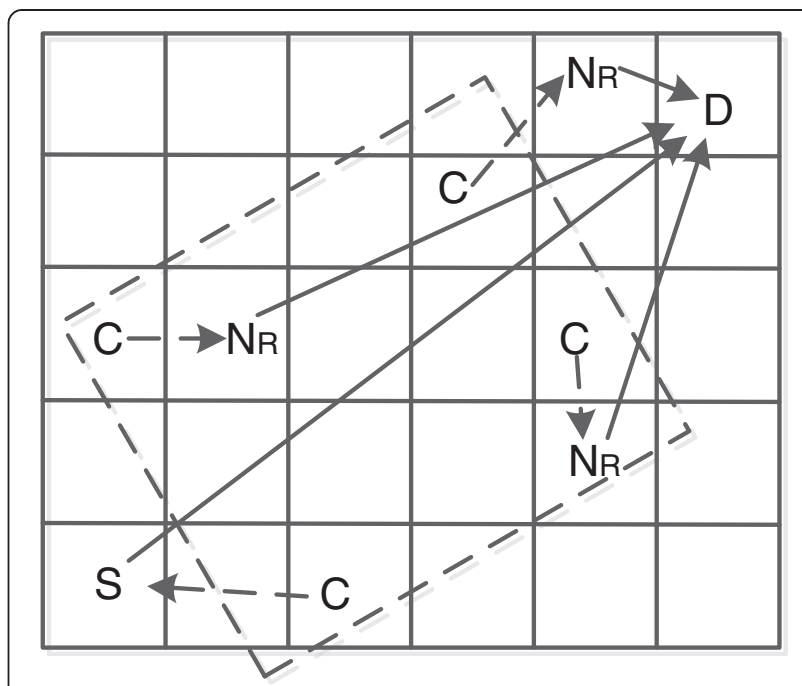

Fig. 5 Topology of the multi-relay cooperative transmission

to the requirements of the user and the real status of the network channel. Then, the $S$ sensor and relay sensors would choose the optimal multimedia clouds for accessing the multimedia stream. Here, let the arrow denote that sender and relay sensors obtain the multimedia streaming data from different clouds.

The third stage: hierarchical cooperative decoding strategy.

In this stage, $D$ sensor could receive the $\frac{m}{n} N_{R} N_{M}$ packets from $S$ sensor and $N_{R}$ relay sensors. The transmission cyclic process of the multi-relay hierarchical cooperative transmission is illustrated in Fig. 6.

\section{Performance evaluation}

In this work, the proposed multimedia cloud, opportunistic dynamic multimedia (ODM) cloud platform, QoS guarantee protocol with the combination of opportunistic dynamic multimedia cloud platform and cooperative multimedia (CM) stream for WSNs (ODM-CM-WSN) are simulated, analyzed, and evaluated with two group experiments, which include the following metrics: average time for running, total time for running, average delivery delay, convergence overhead, parallel efficiency, average delay, outage probability, energy efficiency, and average PSNR.

It is well known that stability, resource utilization rate, computing efficiency, and reliability are four important design goals of cloud computing. And, the real-time performance is the foremost requirement of the multimedia communication. Hence, we designed the experiment 1 for four performance metrics, which was used to compare the proposed multimedia cloud ODM with the public normal cloud platform. The architecture of the experiment 1 is shown in Fig. 7. 


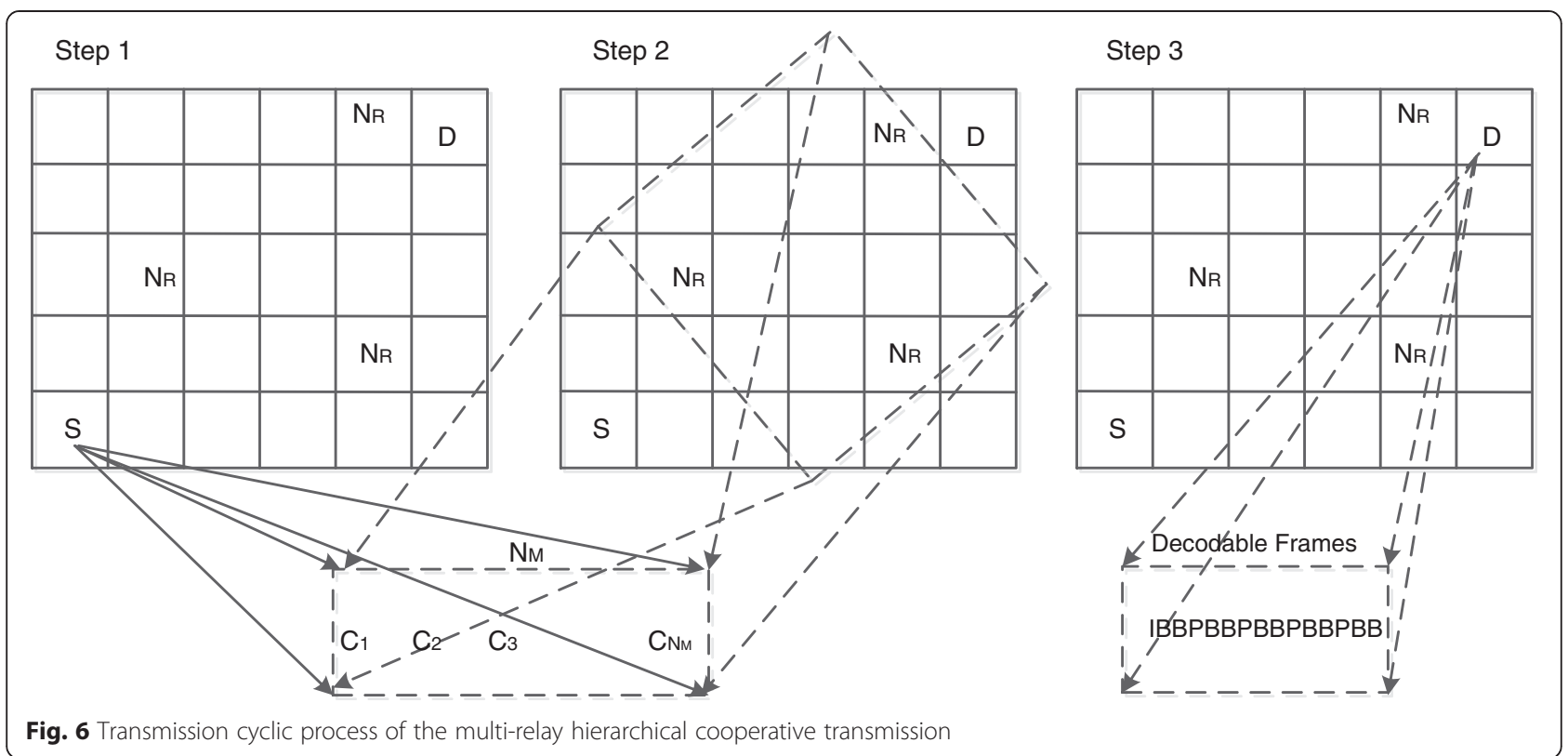

There are two parts in Fig. 7. The first part means the public normal cloud and cloud platform. The clients access the multimedia stream from the cloud platform. The second part means the proposed ODM cloud platform demonstrated in second 2 . We added up and analyzed the average time for running, total time for running, average delivery delay, and convergence overhead at clients, which are illustrated in Figs. 8, 9, 10, 11, and 12.

Figure 8 shows the average time for running of cloud for accessing the multimedia stream from cloud platform

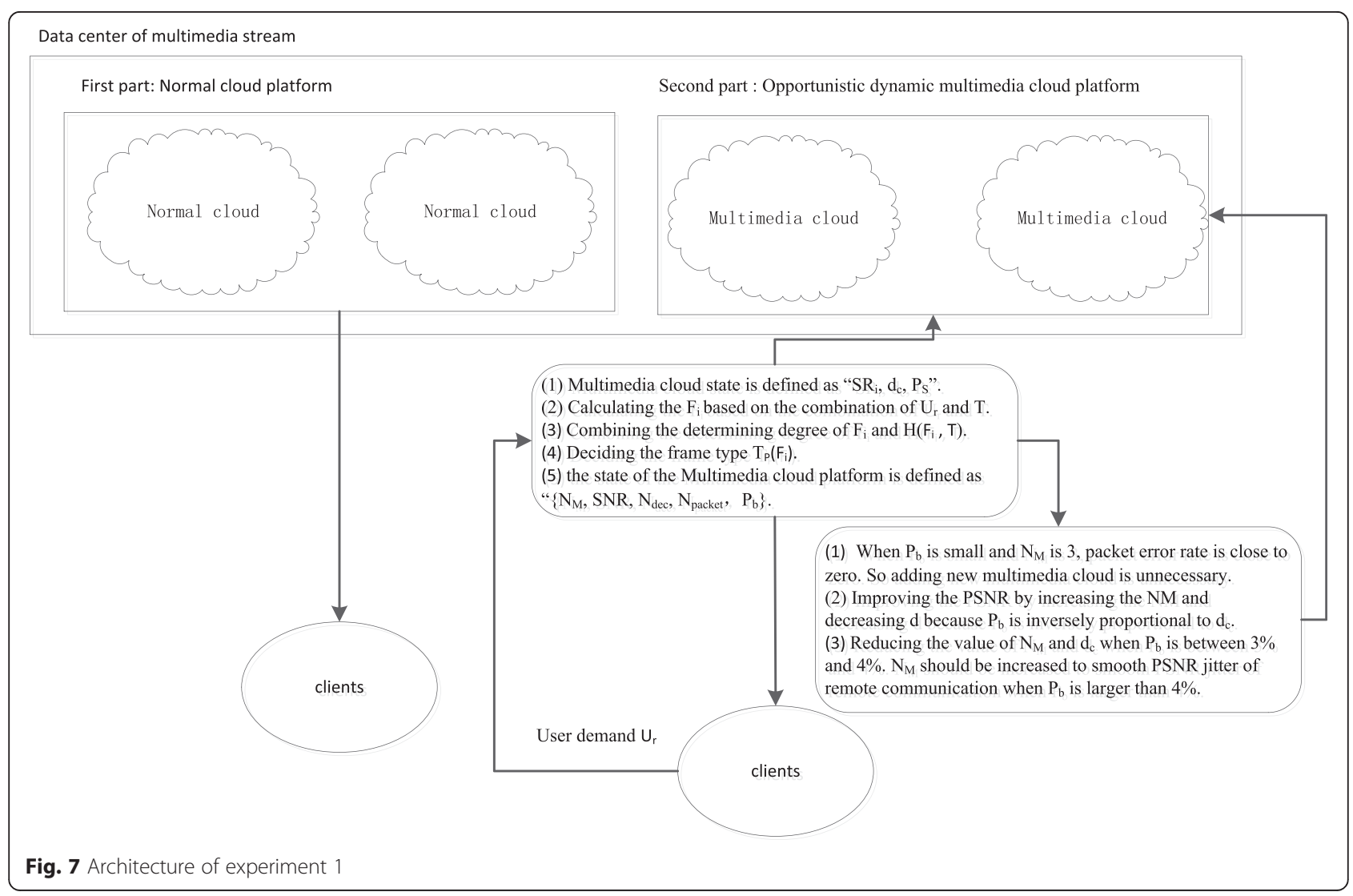




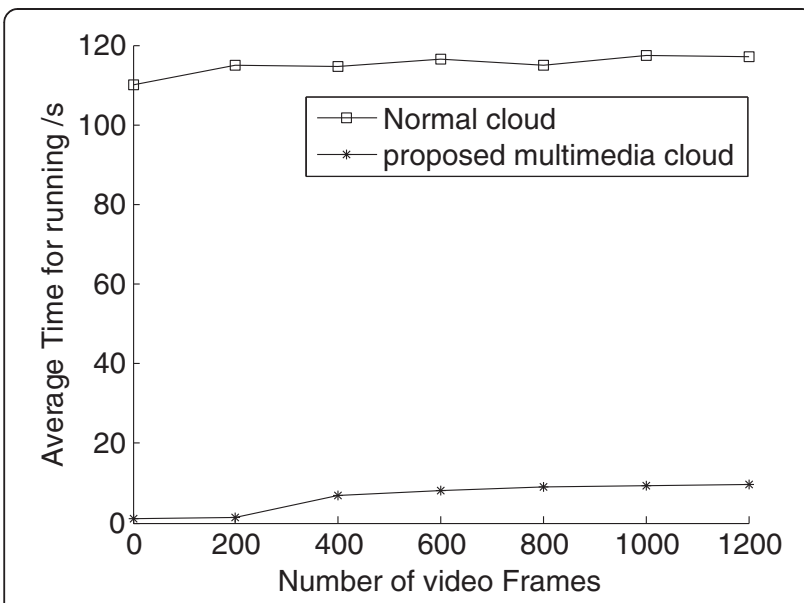

Fig. 8 Average time for running

with number of video frames of the multimedia stream. We found that the time of the proposed multimedia cloud is significantly less than one of that of the normal cloud. The proposed multimedia cloud can reduce the average running time to about $110 \mathrm{~s}$ and the total running time to $50 \mathrm{~s}$ as shown in Fig. 9. That indicates that the proposed multimedia cloud could better support the multimedia application than the normal cloud. That is because the proposed multimedia cloud would find the optimal multimedia resources and invoke the resource based on the type and scale of video frame type, as well as considering the user demand. So, the proposed multimedia could provide the high reliability, real-time, and robust multimedia streaming service.

Figures 10, 11, and 12 show the analytical results of average delivery delay, convergence overhead, and parallel efficiency with number of clouds. The real-time performance of the proposed ODM cloud platform is illustrated in Fig. 10. It was found that the average delivery delay from cloud to clients of the normal cloud platform is larger

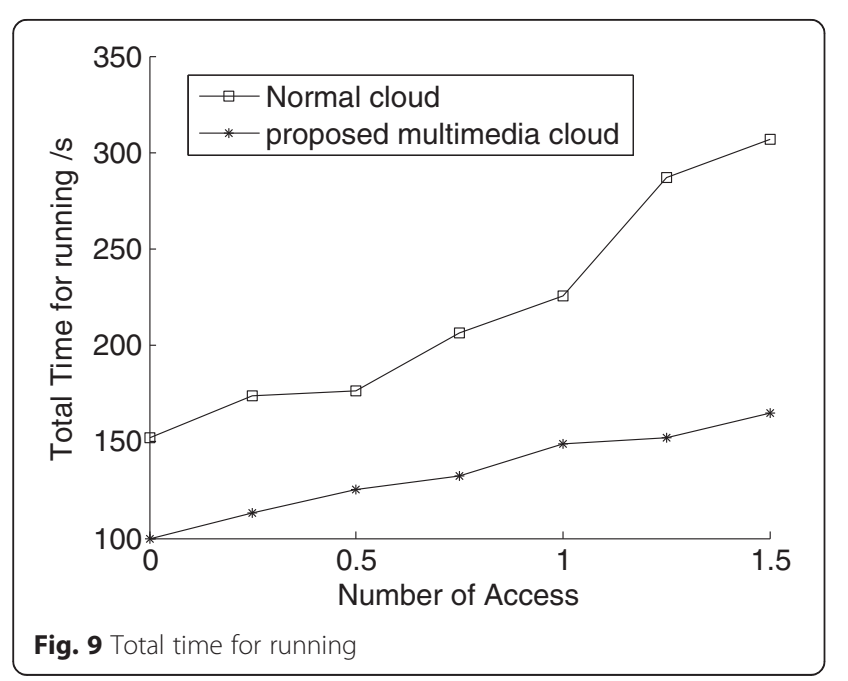

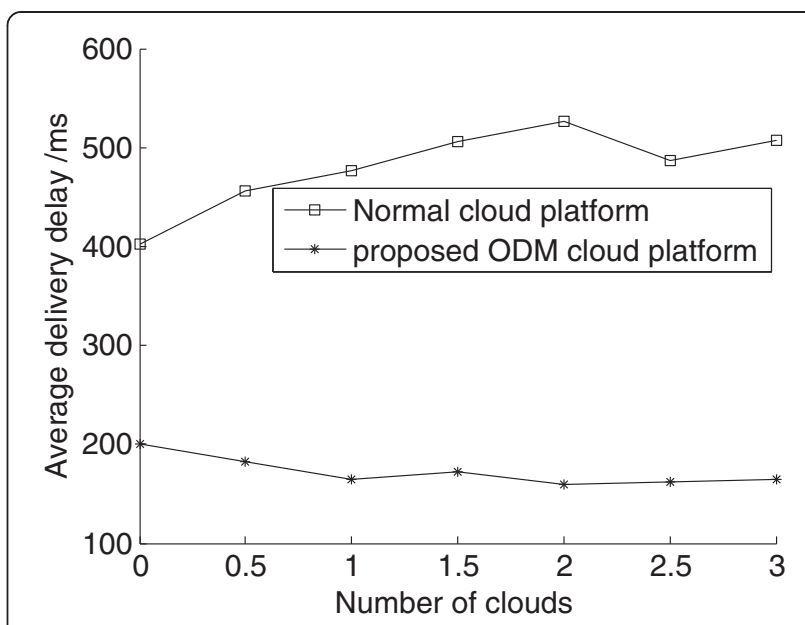

Fig. 10 Average delivery delay with number of clouds

than one of that of the proposed ODM cloud platform. The maximum gap between them is about $317 \mathrm{~s}$. The minimum gap is about $203 \mathrm{~s}$. These declare that the proposed ODM cloud platform has higher capacity of realtime guarantee than the public normal cloud platform. The proposed ODM cloud platform could establish a large-scale multimedia communication infrastructure. The multimedia cloud and opportunistic organization are combined to make it efficient and reliable to operate. The real-time performance of multimedia stream would benefit from multimedia cloud architecture. If one multimedia cloud takes a lot of storage capacity, running time, or delivery time, it is fast and convenient to reconstruct the cloud platform. Figure 11 shows the convergence overheads for the above cloud platform of multimedia stream. There is a sharp drop of iteration with proposed ODM cloud platform when greedy factor increases from 0 to 0.4 . Then, it was observed that a flat small number is less than 15. However, the public normal cloud platform still needs

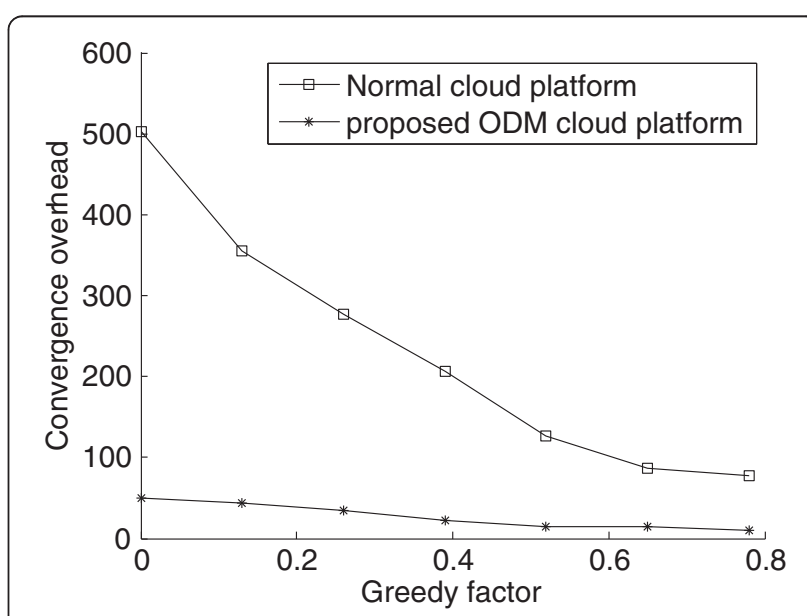

Fig. 11 Convergence overhead with greedy factor 


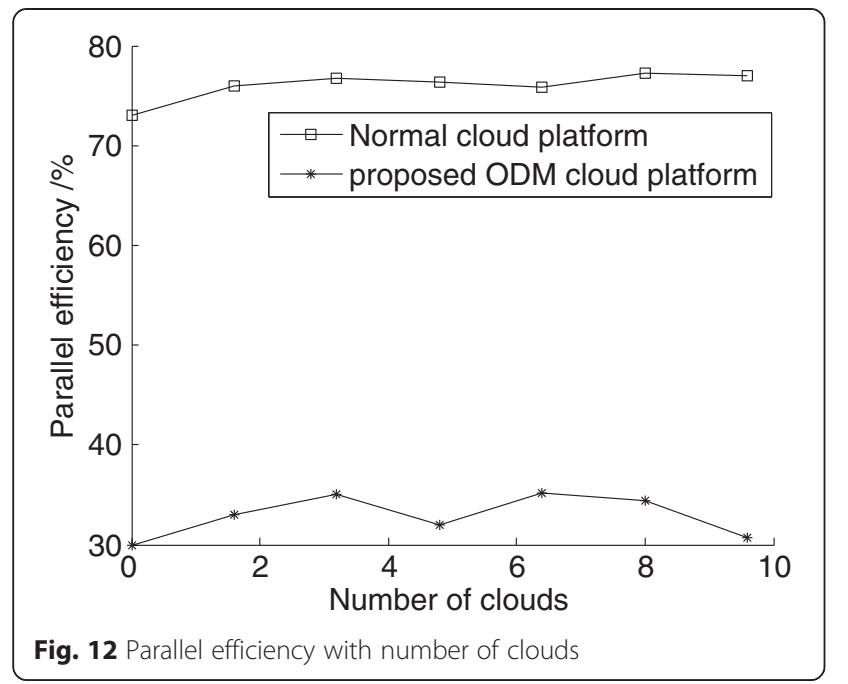

more than 100 iterations to converge, which converges very slowly and cannot support efficient computation shown in Fig. 12. The reduction of computation overhead for multimedia with increase of greedy factor demonstrates a significant improvement of cloud resources and traffic of the cloud platform. As shown in Fig. 12, the proposed ODM cloud platform can share the cloud resource and interact multimedia stream data by sensing the multimedia cloud status and multimedia communication between the clouds. At the same time, the proposed ODM cloud platform can dynamically adjust multimedia cloud platform by opportunistic establishment way, which can provide efficient, real-time, and reliable multimedia cloud services for the clients.
In experiment 2, the proposed ODM-CM-WSN and QoS guarantee protocol based on the normal cloud with WSNs (NC-WSN) were evaluated and studied according to the layered architectural model for multimedia applications as shown in Fig. 13. There are three layers in the multimedia communication model, which are multimedia application layer, cloud platform layer, and infrastructure layer. In the QoS guarantee protocol with NC-WSN, the multimedia stream would be stored in public normal cloud platform, which would be transferred to clients through WSNs with direct communication. However, the multimedia stream of the multimedia application layer would be computed and constructed by the multimedia clouds. The ODM cloud platform could manage the multimedia stream and optimize the interactive interface of the infrastructure layer through the opportunistic dynamic adjustment scheme. Sending sensor or relay sensor would reconstruct the cooperation multimedia stream according to the status of multimedia clouds.

Figure 14 gives the multimedia cloud service of the proposed ODM-CM-WSN based on opportunistic dynamic cloud platform and cooperative multimedia with sensors. The cloud service system can be viewed as a mixed access transmission system with the proposed ODM cloud platform and cooperative multimedia optimal transmission scheme based on the characteristic of multimedia stream, status of cloud platform, and constraint conditions of WSNs. At the beginning of multimedia stream, the ODM cloud platform receives the demand from the clients and reconstructs the multimedia cloud, which includes the real-time performance, reliability, playable quality, etc. Then, the proposed ODM cloud

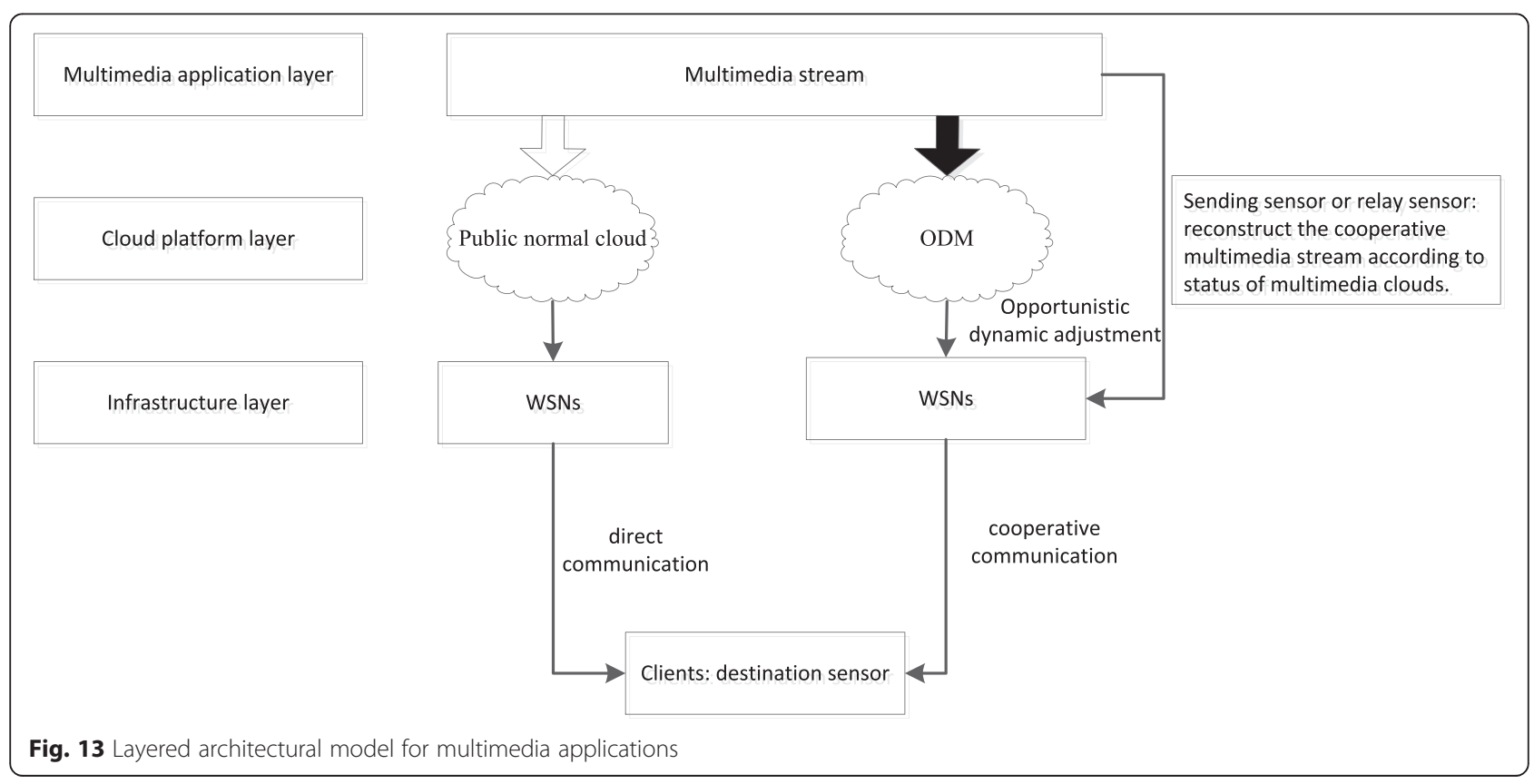




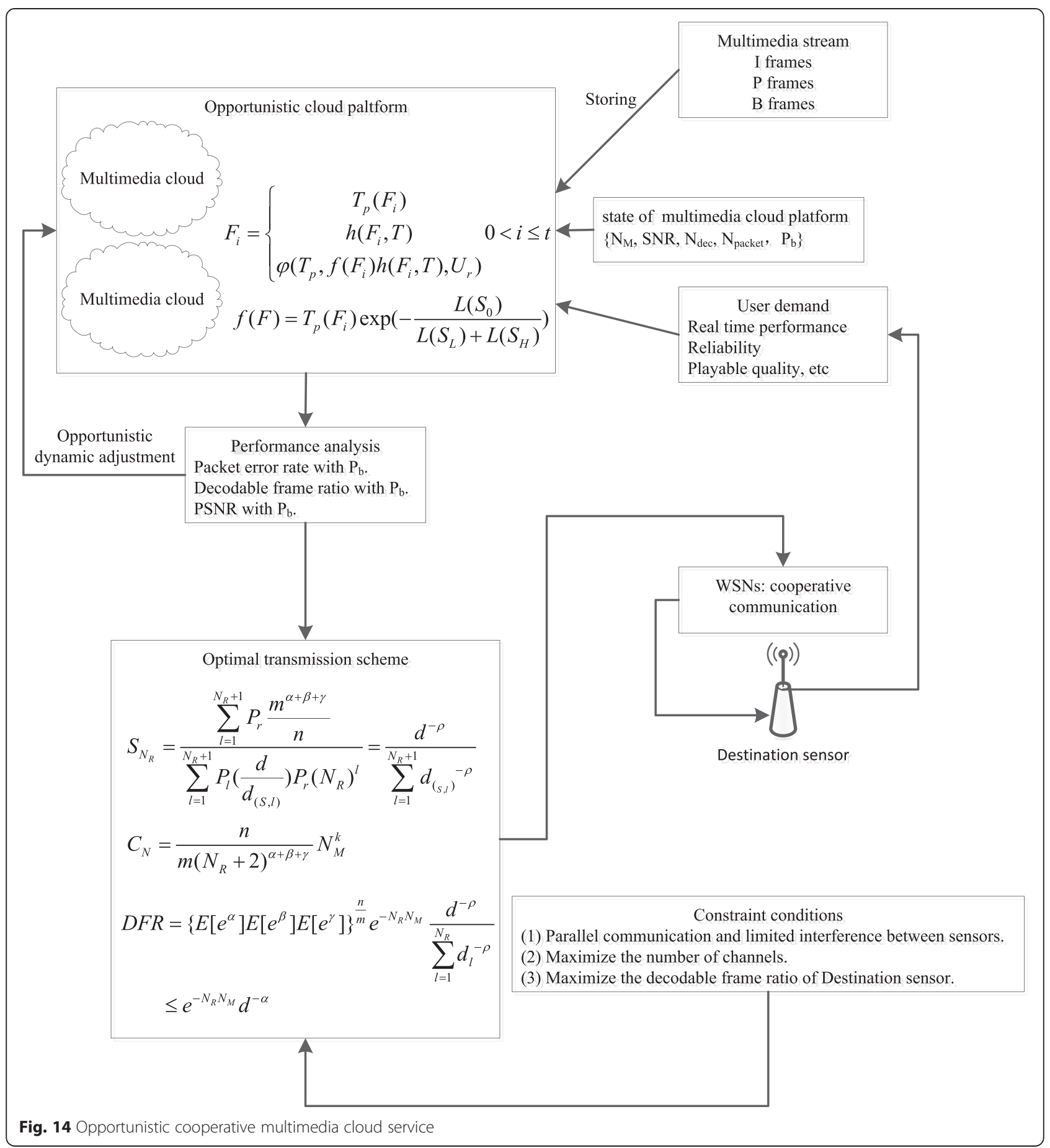

platform is composed by some multimedia clouds with opportunistic scheme based on the analytical results including packet error rate, decodable frame ratio, and PSNR with $P_{b}$.

First, we compare and evaluate the average delay, outage probability, energy efficiency, and average PSNR with distance between sender sensor and receiver sensor, which are shown in Figs. 15, 16, 17, and 18. Figure 15 shows the average end-to-end delay of multimedia stream. It was found that the larger the distance, the larger the delay. Especially, the delay of NC-WSN scheme has a sharp increase when the distance is larger than $4 \mathrm{~km}$. However, the proposed ODM-CM-WSN scheme can smooth the delay jitter. The reliability and stability is illustrated in Fig. 16. When the distance is larger than $4 \mathrm{~km}$, the outage probability of NC-WSN scheme increased 


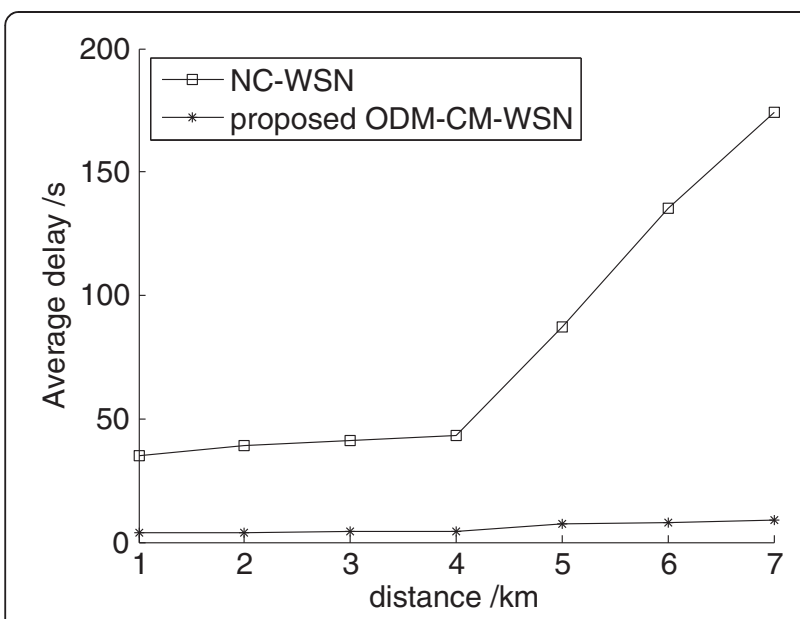

Fig. 15 Average delay with distance

significantly, which demonstrated that the NC-WSN scheme cannot support the multimedia application with long-distance communication. However, the proposed ODM-CM-WSN scheme can optimize the communication scheme by reconstruct the cooperative multimedia stream at infrastructure layer. Figure 17 shows the energy consumption of WSNs based on the two schemes. We found that the lesser the energy efficiency, the larger the distance. However, the energy efficiency of the proposed ODM-CM-WSN scheme is larger than one of that of the NC-WSN scheme, which benefit from the efficient and reliable cloud computing services of multimedia stream and cooperative multimedia architecture. The average PSNR is compared in Fig. 18. There is a sharp drop of NC-WSN scheme with increase of distance because of the unreasonable structure of the multimedia stream for wireless sensor networks. However, average PSNR of proposed ODM-CMWSN scheme decreases slowly and is close to 30 when the distance is larger than $7 \mathrm{~km}$, which benefited from the

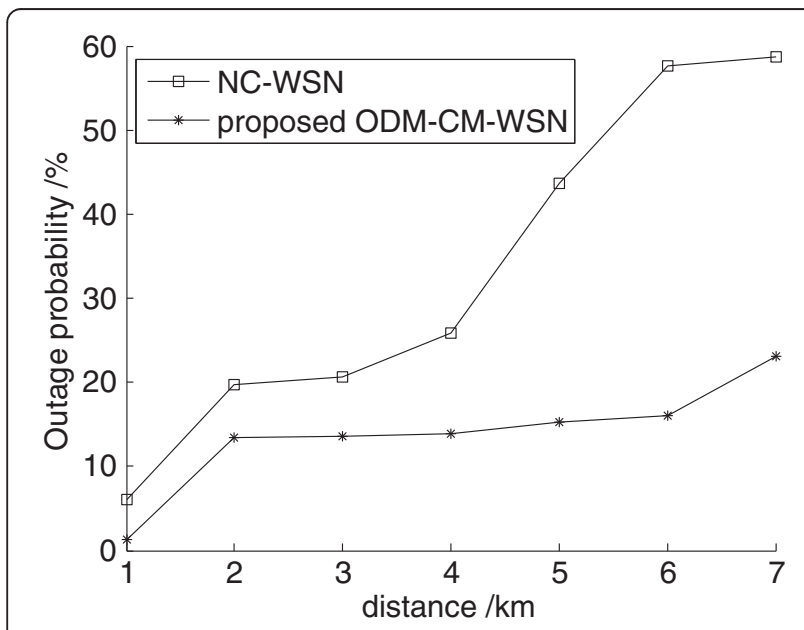

Fig. 16 Outage probability with distance

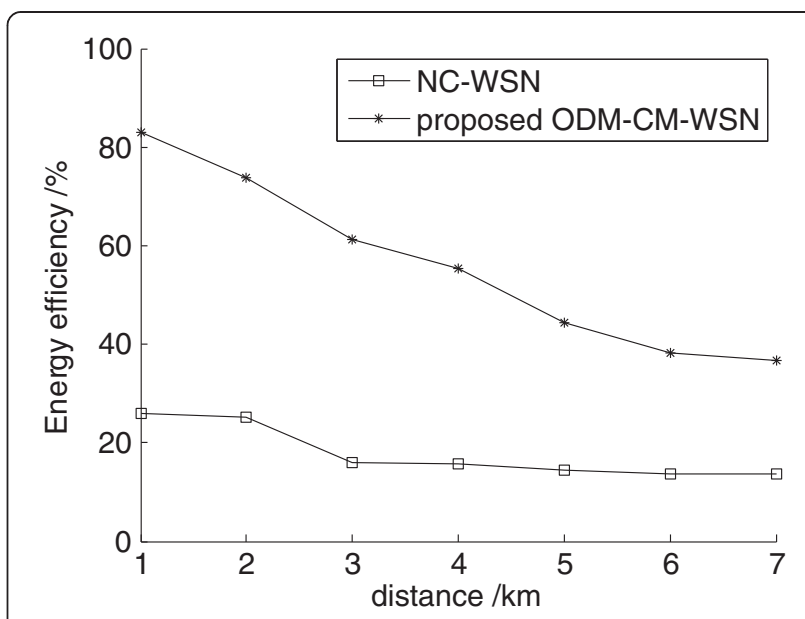

Fig. 17 Energy efficiency with distance

cooperative multimedia streaming structure and dynamical cloud. So, the above results show that the proposed ODM-CM-WSN scheme can guarantee the long-time remote multimedia application service and satisfy the real-time performance, reliability, energy, and playable quality requirements.

Second, the impact of SNR on average delay, outage probability, energy efficiency, and average PSNR is illustrated in Figs. 19, 20, 21, and 22. The better the channel quality and traffic condition, the lesser the average delay as shown in Fig. 19. Specially, the delay of the proposed ODM-CM-WSN scheme is about $30 \mathrm{~s}$ and can improve the real-time performance when the channel is poor. The delay of NC-WSN scheme is about three times one of the proposed ODM-CM-WSN schemes, which cannot support the multimedia stream communication when channel is poor and has worse traffic status than one of the proposed ODM-CM-WSN schemes. As shown in Fig. 20, the two schemes can decrease the outage probability when

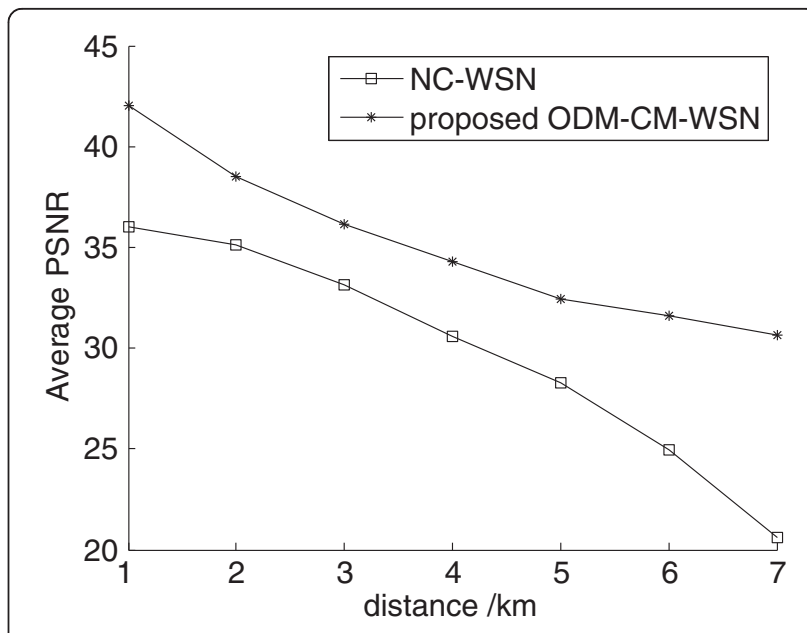

Fig. 18 Average PSNR with distance 


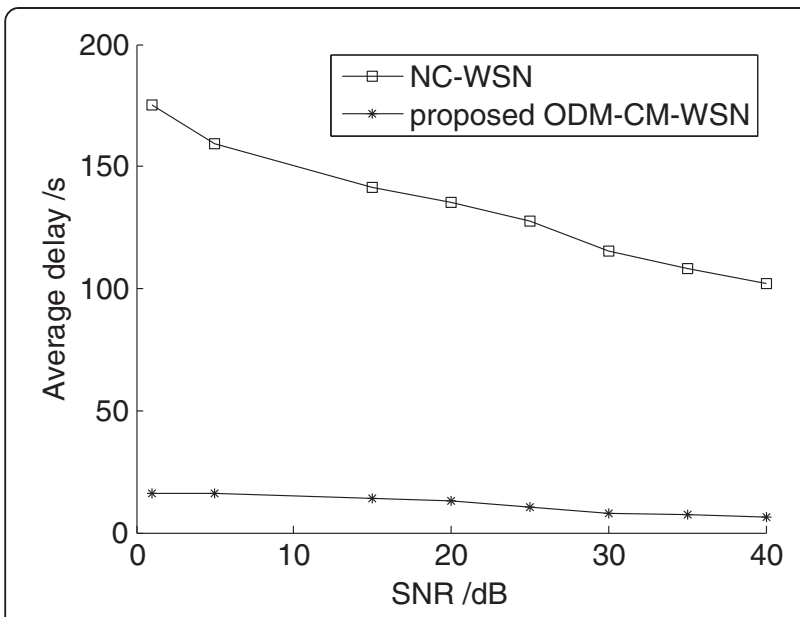

Fig. 19 Average delay with SNR

SNR is increasing. But, the reducing margin and ability of proposed ODM-CM-WSN scheme are superior to one of the NC-WSN schemes. The proposed ODM-CM-WSN scheme has a stronger ability to save energy than the NC-WSN, which benefit from the cooperative multimedia architecture based on the multimedia cloud status and multi-relay hierarchical cooperative transmission scheme. When SNR is larger than $4 \mathrm{~dB}$, the proposed ODM-CM-WSN scheme can raise the average PSNR obviously. However, the average PSNR curve of NCWSN scheme is almost a straight line.

\section{Conclusions}

The brief achievements of this research are as follows. First, we researched the multimedia cloud based on the characteristics of GOP, determining degree, and video frame density, and proposed opportunistic dynamic multimedia cloud platform according to the analytical result of packet error rate, decodable frame ratio, and

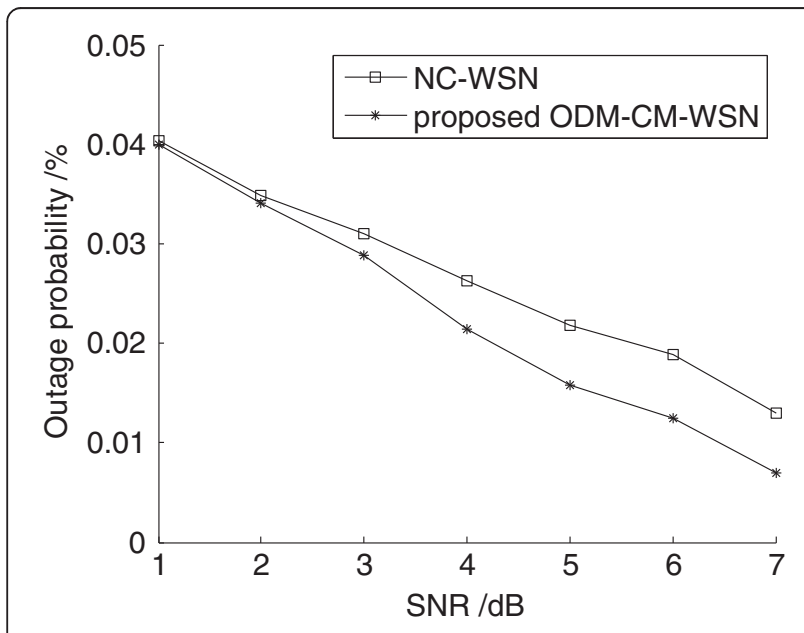

Fig. 20 Outage probability with SNR

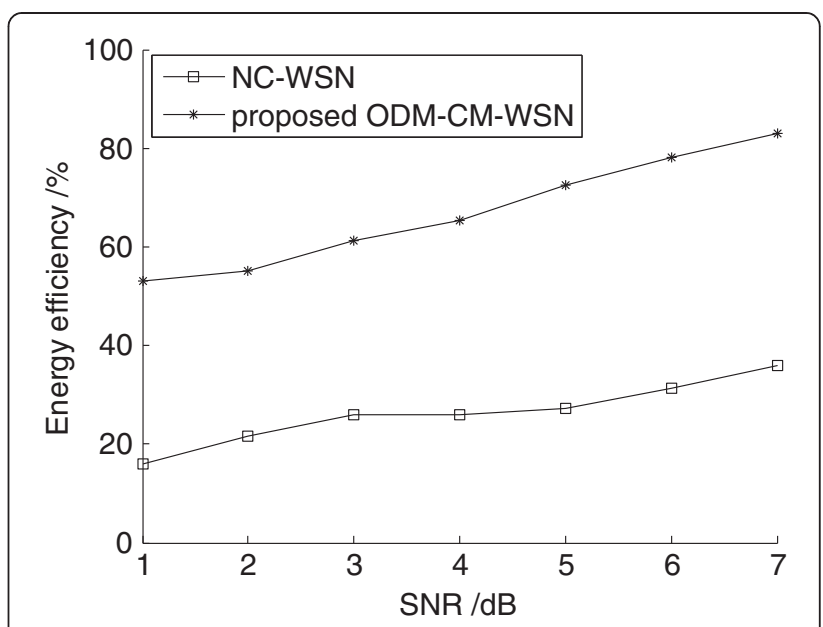

Fig. 21 Energy efficiency with SNR

PSNR. Second, the cooperative multimedia stream reconstruction strategy with the framing of GOP is designed to ensure that infrastructure layer has the optimal multirelay hierarchical cooperative multimedia transmission scheme. Third, combining the opportunistic dynamic multimedia cloud platform and cooperative multimedia stream with WSNs can improve the cloud computing efficiency of multimedia stream and optimize the encoding and decoding complexity, as well as enhance the resources utilization of WSNs. Finally, we present the QoS guarantee protocol with the combination of ODM-CMWSN and the layered architectural model for multimedia applications, as well as the opportunistic cooperative multimedia cloud service model.

The mathematical and simulation results illustrate that the proposed QoS guarantee protocol can achieve the significant gains in terms of average delay, reliability, and throughput, as well as PSNR. As a result, the proposed

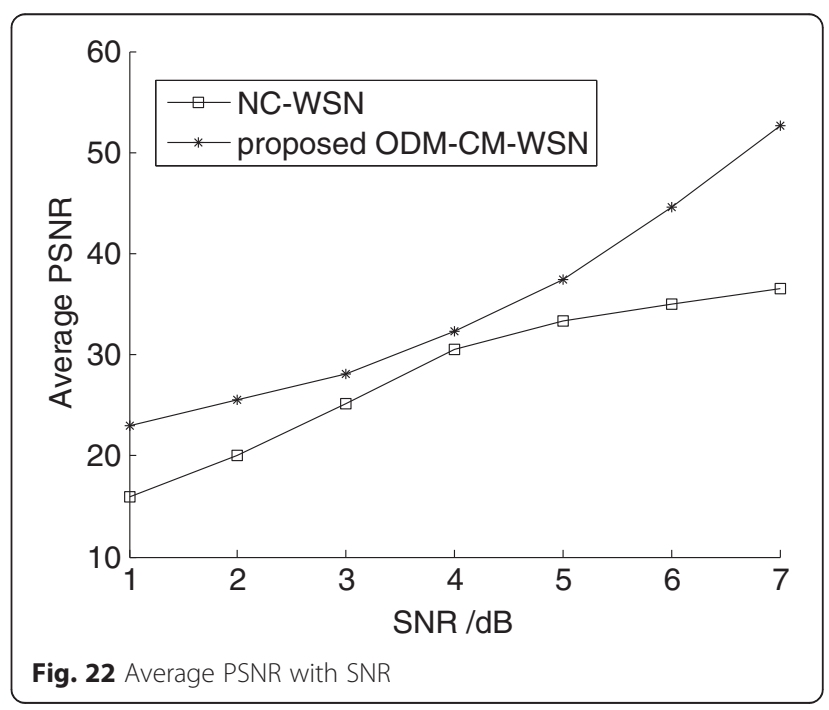


ODM-CM-WSN scheme can satisfy the requirements of multimedia stream application and provide reliable and efficient cloud computing services with WSNs.

\section{Competing interests}

The authors declare that they have no competing interests.

\section{Acknowledgements}

This work is supported in part by the science and technology development project of Changshu (Social Development category) grant No. CS201413, National Natural Science Foundation of China grant No. 61300186, and Project of Jiangsu Province University Natural Science grant No. 13KJB510001.

\section{Author details}

${ }^{1}$ School of Computer Science \& Engineering, Changshu Institute of Technology, Changshu 215500, China. ${ }^{2}$ Changshu Museum, Changshu 215500, China. ${ }^{3}$ College of Computer Science and Technology, Taiyuan University of Technology, Taiyuan 030024, China.

Received: 10 February 2015 Accepted: 3 June 2015

Published online: 11 June 2015

\section{References}

1. S Park, SJ Kwon, A study on factors affecting intention to switch for using cloud computing: a case of Google Docs. J. Inf. Tech. Serv 10(3), 149-166 (2011)

2. JH Yoon, A study of the systems quality effect on the intention to use of cloud computing services in information center. Journal of the Korean Society for Information Management 28(4), 49-63 (2011)

3. Y Xu, S Mao, A survey of mobile cloud computing for rich media applications. IEEE Wirel. Commun. 20(3), 46-53 (2013)

4. SS Manvi, GK Shyam, Resource management for infrastructure as a service (laaS) in cloud computing: a survey. J. Netw. Comput. Appl. 41, 424-440 (2014)

5. AN Khan, MLM Kiah, SU Khan et al., Towards secure mobile cloud computing: a survey. Future generation computer systems. The International Journal of Grid Computing and eScience 29(5), 1278-1299 (2013)

6. MD Ryan, Cloud computing security: the scientific challenge, and a survey of solutions. J. Syst. Softw. 86(9), 2263-2268 (2013)

7. A Alamri, WS Ansari, MM Hassan et al., A survey on sensor-cloud: architecture, applications, and approaches. International Journal of Distributed Sensor Networks 2013(917923), 18 (2013)

8. LQ Zeng, QW Han, X Wu et al., Abnormal vehicle oriented infrastructure based on wireless sensor network and cloud computing. Sens. Lett. 11(5), 918-925 (2013)

9. MR Rahimi, J Ren, CH Liu et al., Mobile cloud computing: a survey, state of art and future directions. Mobile networks \& applications 19(2), 133-143 (2014)

10. M Choi, J Park, YS Jeong. J. Supercomput. 64(2), 331-356 (2013)

11. C-H Lin, P Hsiu, C-K Hsieh, Dynamic backlight scaling optimization: a cloud-based energy-saving service for mobile streaming applications. IEEE Trans. Comput. 63((2), 335-347 (2014)

12. I Mehmood, M Sajjad, SW Baik, Mobile-cloud assisted video summarization framework for efficient management of remote sensing data generated by wireless capsule sensors. Sensors 14(9), 17112-17145 (2014)

13. AH Farooqi, FA Khan, J Wang et al., A novel intrusion detection framework for wireless sensor networks. Pers. Ubiquit. Comput. 17(5), 907-919 (2013)

14. Q Zhou, DQ Xiao, Y Tang et al., Trusted big data capture and transport architecture for wireless sensor network. J. Internet Technol. 15(6), 1033-1041 (2014)

15. O Diallo, JJPC Rodrigues, M Sene et al., Real-time query processing optimization for cloud-based wireless body area networks. Inf. Sci. 284, 84-94 (2014)

16. B Li, B Qi, Y Sun et al., Fault-tolerant energy efficiency computing approach for sparse sampling under wireless sensor smart grid. Wirel. Pers. Commun. 79(3), 2041-2058 (2014)

17. S Shamshirband, NB Anuar, MLM Kiah et al., An appraisal and design of a multi-agent system based cooperative wireless intrusion detection computational intelligence technique. Eng. Appl. Artif. Intell. 26(9), 2105-2127 (2013)

18. M Garcia-Valls, T Cucinotta, CY Lu, Challenges in real-time virtualization and predictable cloud computing. J. Syst. Archit. 60(9), 726-740 (2014)
19. D Kourtesis, JM Alvarez-Rodriguez, I Paraskakis, Semantic-based QoS management in cloud systems: current status and future challenges. Future Generation Computer Systems-The International Journal of Grid Computing and escience 32, 307-323 (2014)

20. LDP Mendes, JJPC Rodrigues, J Lloret, LDP Mendes, JJPC Rodrigues, J Lloret et al., Cross-layer dynamic admission control for cloud-based multimedia sensor networks. IEEE Syst. J. 8(1), 235-246 (2014)

21. S Hussain, JH Bang, M Han et al., Behavior life style analysis for mobile sensory data in cloud computing through MapReduce. Sensors 14(11), 22001-22020 (2014)

22. S Misra, S Chatterjee, Social choice considerations in cloud-assisted WBAN architecture for post-disaster healthcare: data aggregation and channelization. Inf. Sci. 284, 95-117 (2014)

23. R Dautov, I Paraskakis, M Stannett, Towards a framework for monitoring cloud application platforms as sensor networks. Cluster computing-the journal of networks software tools and applications 17(4), 1203-1213 (2014)

24. CS Zhu, H Nicanfar, VCM Leung et al., An authenticated trust and reputation calculation and management system for cloud and sensor networks integration. IEEE Transactions on Information Forensics and Security 10(1), 118-131 (2015)

25. SL Ren, M van der Schaar, Efficient resource provisioning and rate selection for stream mining in a community cloud. IEEE Transactions on Multimedia 15(4), $723-734$ (2013)

\section{Submit your manuscript to a SpringerOpen ${ }^{\odot}$ journal and benefit from:}

- Convenient online submission

- Rigorous peer review

- Immediate publication on acceptance

- Open access: articles freely available online

- High visibility within the field

- Retaining the copyright to your article

Submit your next manuscript at springeropen.com 